\title{
Synergistic intrafibrillar/extrafibrillar mineralization of collagen scaffolds based on a biomimetic strategy to promote the regeneration of bone defects
}

\author{
This article was published in the following Dove Press journal: \\ International Journal of Nanomedicine \\ 12 May 2016 \\ Number of times this article has been viewed
}

\author{
Yao Wang \\ Ngo Van Manh ${ }^{1,2}$ \\ Haorong Wang' \\ Xue Zhong' \\ Xu Zhang' \\ Changyi Li' \\ 'School of Dentistry, Hospital \\ of Stomatology, Tianjin Medical \\ University, Tianjin, People's Republic \\ of China; ${ }^{2}$ Thaibinh University of \\ Medicine and Pharmacy, Thaibinh, \\ Vietnam
}

\begin{abstract}
The mineralization of collagen scaffolds can improve their mechanical properties and biocompatibility, thereby providing an appropriate microenvironment for bone regeneration. The primary purpose of the present study is to fabricate a synergistically intra- and extrafibrillar mineralized collagen scaffold, which has many advantages in terms of biocompatibility, biomechanical properties, and further osteogenic potential. In this study, mineralized collagen scaffolds were fabricated using a traditional mineralization method (ie, immersed in simulated body fluid) as a control group and using a biomimetic method based on the polymer-induced liquid precursor process as an experimental group. In the polymer-induced liquid precursor process, a negatively charged polymer, carboxymethyl chitosan (CMC), was used to stabilize amorphous calcium phosphate (ACP) to form nanocomplexes of CMC/ACP. Collagen scaffolds mineralized based on the polymer-induced liquid precursor process were in gel form such that nanocomplexes of $\mathrm{CMC} / \mathrm{ACP}$ can easily be drawn into the interstices of the collagen fibrils. Scanning electron microscopy and transmission electron microscopy were used to examine the porous micromorphology and synergistic mineralization pattern of the collagen scaffolds. Compared with simulated body fluid, nanocomplexes of CMC/ACP significantly increased the modulus of the collagen scaffolds. The results of in vitro experiments showed that the cell count and differentiated degrees in the experimental group were higher than those in the control group. Histological staining and micro-computed tomography showed that the amount of new bone regenerated in the experimental group was larger than that in the control group. The biomimetic mineralization will assist us in fabricating a novel collagen scaffold for clinical applications.
\end{abstract}

Keywords: collagen scaffold, intrafibrillar mineralization, extrafibrillar mineralization, tissue engineering, nanocomplexes

\section{Introduction}

Although autografts and allografts have been used to reconstruct bone defects caused by tumor resections or traumas in clinic, significant drawbacks still remain, including the morbidity of the donor site, the limited volume of available bone, the host's immunoreactions, and even some infectious diseases. Thus, repairing large bone defects continues to be a challenge for bone regeneration. With the development of bone tissue engineering, scaffolds fabricated using different biomaterials, such as bioactive glasses, polylactic acid, collagen, chitosan, and hydroxyapatite (HA), have been explored for bone regeneration due to their biocompatibility, sufficient supply, and no secondary damage for patients. ${ }^{1-7}$ Among these biomaterials, type 1
Correspondence: Xu Zhang; Changyi Li School of Dentistry, Hospital of Stomatology, Tianjin Medical University, 12 Observatory Road, Tianjin 300070 People's Republic of China Email zhangxu@tmu.edu.cn; changyi_li@sina.com 
collagen and HA, as the predominant organic and inorganic components of bone extracellular matrix (ECM), are always considered to be the ideal materials for scaffolds. ${ }^{5}$ Natural bone tissue is a complex hierarchical system of synergistically intra- and extrafibrillar mineralized collagen fibers. ${ }^{1}$ The mineral phase within bone and dentin can be divided into two primary existing forms: intrafibrillar mineralization, which is located inside or near the gap zones of collagen microfibrils and stretches along the long axis of the fibrils, and extrafibrillar mineralization, which is deposited outside the collagen fibrils. ${ }^{8}$ Therefore, an ideal collagen scaffold should not only mimic the native bone ECM in composition but also reproduce its microstructure of intra- and extrafibrillarly mineralized collagen fibers. ${ }^{9}$

To date, mixing collagen with minerals and mineralizing collagen have been used to modify collagen scaffolds for simulating the composition and microstructure of bone tissue to the greatest extent possible. ${ }^{10-17}$ Because HA is a primary mineral component of natural bone and possesses outstanding bioactivity, osteoconductivity, biocompatibility with bone cells, good mechanical properties, and a slow degradation rate in vivo, it has often been the first choice for modifying collagen scaffolds. ${ }^{10,18-20}$ Although directly mixing collagen with HA powders in certain proportions can result in collagen scaffolds with compositions similar to those of natural bone tissues, it is difficult to simulate their microstructure and microenvironment via this process. Therefore, to solve the aforementioned problems, researchers are devoting more attention to the biomimetic mineralization of collagen scaffolds. ${ }^{12,16,17,21-23}$ The aforementioned works primarily focus on depositing HA crystals outside the collagen fibrils using traditional mineralization methods, such as immersing the collagen scaffolds in different modified simulated body fluids (SBFs). This process is called extrafibrillar mineralization..$^{9,23,24}$ Although extrafibrillar mineralization can produce composite scaffolds that are similar to natural bone ECM in terms of chemical composition and a small fraction of structures, it is incapable of simulating the nanoscale hierarchical self-assembled structure in the mineralized collagen fibrils of natural bone. ${ }^{9}$ More recently, some researchers have demonstrated that collagen scaffolds mineralized using a polymer-induced liquid precursor (PILP) mineralization process based on nonclassical crystallization theory can achieve a bone-like nanostructure with HA nanocrystals embedded within collagen fibrils. The biomimetic mineralization through this process is called intrafibrillar mineralization..$^{8,9,14,25-27}$ In contrast to the classical crystallization pathways, the nonclassical crystallization route involves mesoscopic transformations of self-assembled, metastable, or amorphous precursor particles into nanoparticulate superstructures. ${ }^{28,29}$ Because this process assembles materials from the nanoscopic scale to large sizes, it is also called the bottom-up strategy. ${ }^{8,30}$ Many previous experiments and studies have shown that biomineralization is a bottom-up process based on the theory of nonclassical crystallization. ${ }^{8,28,31}$ The key of the PILP process is to find negatively charged polymers for stabilizing amorphous precursor particles to achieve liquid-liquid phase separation. Many stabilizers have been used in previous experiments, such as polyaspartic acid, polyacrylic acid, poly-L-glutamic acid, etc, which are analogs of dentine matrix protein-1 (DMP-1). ${ }^{25,32-34}$ DMP-1 is an important acidic noncollagenous protein delivered at the beginning of mineralized matrix formation in bone and dentine. It has been demonstrated that DMP-1 can stabilize amorphous calcium phosphate (ACP) nanoclusters to form nanocomplexes of DMP-1/ACP by self-assembly, revealing special functions during the mineralization in the gap zone of collagen. ${ }^{35-38}$ The minor phase formed in this process is composed of metastable, amorphous, liquid-phase nanodroplets, which are the precursors of ACP. ${ }^{25}$ It has been proposed that these precursors can be drawn into the interstices of the collagen fibrils by capillary forces of fluidic nature. Subsequently, due to the transformation of ACP to HA, collagen fibrils are embedded with HA nanocrystals similar to the natural bone structure. ${ }^{39,40}$

Based on this bionic concept, we have found a new type of analog called chitosan, which is derived from the shells of shellfish and natural polysaccharides of some fungal cell walls. It has been reported that carboxymethyl chitosan (CMC), the derivative of chitosan enriched in carboxyl groups, can retard or inhibit the rate of spontaneous calcium phosphate precipitation. ${ }^{41}$ In our previous study, we demonstrated that CMC can stabilize ACP to form liquid-phase nanocomplexes of CMC/ACP, which can aid in intrafibrillar mineralization of collagen using the bottom-up strategy based on nonclassical crystallization theory, thereby facilitating the remineralization of demineralized dentine. ${ }^{37}$ Therefore, this prompted us to consider using CMC to imitate the function of the important noncollagenous protein, dentin matrix protein, and then biomimetically mineralize the type 1 collagen scaffolds for bone tissue regeneration.

The objective of the present study was to manufacture a mineralized three-dimensional biomimetic mineralized collagen scaffold (BMC) based on biomimetic theory and to compare it with a non-mineralized collagen scaffold (NMC) and traditional mineralized collagen scaffold (TMC) by SBF 
in terms of morphology, mechanical properties, and biological properties through in vivo and in vitro experiments. The hypothesis of this study was that the collagen scaffold can undergo synergistic intra- and extrafibrillar mineralization by the nanocomplexes of CMC/ACP based on PILP theory. The BMC scaffold, which was similar to natural bone ECM in terms of chemical composition and microstructure, can better promote the regeneration of bone in defects.

\section{Materials and methods}

\section{Fabrication of BMC, TMC, and NMC scaffolds}

The modified SBF was prepared by dissolving $\mathrm{NaCl}$ (7.996 g/L), $\mathrm{NaHCO}_{3}(0.350 \mathrm{~g} / \mathrm{L}), \mathrm{KCl}(0.244 \mathrm{~g} / \mathrm{L})$, $\mathrm{K}_{2} \mathrm{HPO}_{4} \cdot 3 \mathrm{H}_{2} \mathrm{O}(0.288 \mathrm{~g} / \mathrm{L}), \mathrm{MgCl}_{2} \cdot 6 \mathrm{H}_{2} \mathrm{O}(0.305 \mathrm{~g} / \mathrm{L}), \mathrm{CaCl}_{2}$ $(0.278 \mathrm{~g} / \mathrm{L}), \mathrm{Na}_{2} \mathrm{SO}_{4}(0.071 \mathrm{~g} / \mathrm{L})$, and tris(hydroxymethyl) aminomethane $(6.057 \mathrm{~g} / \mathrm{L})$ in distilled water as described previously by Shakir et al. ${ }^{42}$ The $\mathrm{pH}$ was adjusted to 7.4 using $1 \mathrm{~mol} / \mathrm{L} \mathrm{HCl}$ at room temperature. The $\mathrm{CMC} / \mathrm{ACP}$ mineral solution was prepared by mixing $1 \% \mathrm{CMC}$ into $40 \mathrm{~mL}$ of water under stirring $(1,000 \mathrm{rpm})$ until the $\mathrm{CMC}$ powder was completely dissolved. Then, $0.052 \mathrm{~g}$ of $\mathrm{K}_{2} \mathrm{HPO}_{4}$ was added to the $\mathrm{CMC}$ solution under stirring (500 rpm). Next, $0.074 \mathrm{~g}$ of $\mathrm{CaCl}_{2}$ was added to $10 \mathrm{~mL}$ of deionized water, and this solution was added dropwise into the CMC solution under stirring (500 rpm) for 5 minutes to form the CMC/ACP solution. The final concentrations of calcium and phosphate ions were $10 \mathrm{mM}$ and $6 \mathrm{mM}$, respectively.

The method for preparing type 1 collagen used in this experiment was modified according to that of Price. ${ }^{43}$ Rat tail tendons from Sprague Dawley (SD) rats were dissolved in acetic acid $(0.03 \mathrm{~mol} / \mathrm{L})$ for 3 days, and then the collagen solution was centrifuged in a refrigerated centrifuge $\left(4^{\circ} \mathrm{C}, 3,000 \mathrm{rpm}\right.$, 30 minutes). The supernatant was placed in dialysis bags to dialyze for 3-5 days in dipotassium phosphate solution (0.02 $\mathrm{mol} / \mathrm{L})$. Then, the collagen hydrogel collected from the dialysis bags was placed in deionized water, SBF, or CMC/ACP mineral solution for a period of time, followed by lyophilization.

\section{Characterization of BMC,TMC, and NMC scaffolds}

\section{Scanning electron microscopy}

The micromorphology of the scaffolds was observed using a scanning electron microscope (SEM; Nova NanoSEM 430; FEI, Hillsboro, OR, USA). Prior to the observations, the samples were sputter-coated with gold under an argon atmosphere using a sputter coater (K575XD, Emitech; Quorum Technologies Ltd., East Sussex, UK).

\section{X-ray diffraction analysis}

$\mathrm{X}$-ray diffraction (XRD) patterns were used to determine the mineral phases of the samples. The samples were scanned with $\mathrm{Cu} \mathrm{K}_{\alpha} \mathrm{X}$-ray radiation at $40 \mathrm{kV}$ and $20 \mathrm{~mA}$ over the $2 \theta$ range of $10^{\circ}-60^{\circ}$.

\section{Swelling tests}

The three different scaffolds were weighed after lyophilization at dry condition $\left(W_{\mathrm{d}}\right)$. Then the scaffolds were soaked in normal saline for $10,20,30,40,50,60,70$, and 80 minutes at $37^{\circ} \mathrm{C}$ and weighed at different time points $\left(W_{\mathrm{w}}\right)$. The swelling ratio was calculated using the formula:

$$
\text { Swelling ratio }=\frac{W_{\mathrm{w}}-W_{\mathrm{d}}}{W_{\mathrm{d}}} \times 100 \%
$$

Each experiment was repeated three times.

\section{Mechanical properties}

The three different scaffolds were prepared into definite specimens ( $2 \mathrm{~mm}$ in thickness, $8 \mathrm{~mm}$ in diameter) for tensile strength measurements. The testing results were obtained using an ultimate tensile test machine (3367; Instron, Norwood, MA, USA) with a crosshead speed of $5 \mathrm{~mm} / \mathrm{min}$ under both wet (soaking in normal saline for 0.5 hours in advance) and dry conditions. Subsequently, the elastic moduli of the specimens were computed and recorded using a Bluehill System (Instron, Norwood, MA, USA).

\section{Biodegradation of the scaffold in vitro}

The different scaffolds were immersed in $1 \mathrm{~mol} / \mathrm{L}$ phosphatebuffered saline (PBS; pH 7.4) with lysozyme $(0.5 \mathrm{mg} / \mathrm{mL}$; Sigma-Aldrich, St Louis, MO, USA). They were incubated under sterile conditions at $37^{\circ} \mathrm{C}$. The solution was replaced every 3 days with fresh medium. Samples were taken out from the medium at different testing points, washed with distilled water, lyophilized, and weighed. The extent of degradation was calculated using the equation: $D=\left(W-W_{0}\right) /$ $W_{0} \times 100 \%$, where $D$ represents mass loss, $W_{0}$ is the initial weight of different scaffolds, and $W$ is the weight after degradation for $3,5,7,14,21$, and 28 days. Each experiment was repeated three times.

\section{Quantitative analysis of minerals within mineralized scaffolds}

The three different kinds of samples of the same quality were divided into dry group and wet group. Afterward, the different kinds of samples in dry group were soaked in 5.25\% sodium hypochlorite with sufficient shock. Next, the solution 
was centrifuged (3,000 rpm, 15 minutes). The precipitate was lyophilized for weighing. After soaking in normal saline for 0.5 hours, the samples in wet group were processed in the same way as the samples in dry group. The mineral content of each specimen was calculated by the formula:

$$
\text { Mineral content }=\frac{M_{1}-M_{0}}{M_{0}} \times 100 \%
$$

where $M_{0}$ is initial weight of different samples and $M_{1}$ is the weight after being dissolved by sodium hypochlorite. Each experiment was repeated three times.

\section{Transmission electron microscopy and selected-area electron diffraction}

Transmission electron microscopy (TEM) was performed to investigate the intra- and extrafibrillar mineralization of different scaffolds. Briefly, 2\% glutaraldehyde was used to fix the specimens. Then, the specimens were dehydrated in an ascending ethanol series from $50 \%$ to $100 \%$. Subsequently, they were immersed in propylene oxide and embedded in epoxy resin. Ultrathin sections were examined using a TEM (JEOL, Tokyo, Japan) operating at $180 \mathrm{kV}$. Selected-area electron diffraction patterns were used to determine the crystallinity of the specimens.

\section{Cell and incubation conditions}

MC3T3-E1 osteoblasts were cultured in Dulbecco's Modified Eagle's Medium (DMEM) containing 10\% fetal bovine serum, $100 \mathrm{mg} / \mathrm{mL}$ streptomycin, and $100 \mathrm{U} / \mathrm{mL}$ penicillin. The three types of scaffolds were cut into discs of diameter $15 \mathrm{~mm}$ to match the well size of a 24-well plate and then sterilized using gamma rays (cobalt-60) at a dose of $25 \mathrm{kGy}$ (Institute of Radiation Medicine, Chinese Academy of Medical Sciences and Peking Union Medical College, People's Republic of China). These sterilized scaffolds were prewetted with complete growth medium (DMEM with 10\% fetal bovine serum, $100 \mathrm{mg} / \mathrm{mL}$ streptomycin, and $100 \mathrm{U} / \mathrm{mL}$ penicillin) prior to adding osteoblasts. Then, 10,000 osteoblasts per $1,000 \mu \mathrm{L}$ of medium were seeded onto each sterilized scaffold placed in a new 24-well plate, which were maintained in a $5 \% \mathrm{CO}_{2}$ incubator at $37^{\circ} \mathrm{C}$ for various culturing intervals (ie, 1,3, 5, and 7 days).

\section{Cell assays}

\section{Live/dead cell double staining and} immunofluorescent staining

To observe the distribution and morphology of cells seeded on each scaffold, live/dead cell double staining and immunofluorescent staining were carried out. The different scaffolds were removed from the 24-well plates after 1, 3, 5, and 7 days, washed three times with PBS, placed in new plates, and then stained with an acridine orange/ethidium bromide solution (100 mL per well). For immunofluorescent staining, the cells were fixed with $4 \%$ paraformaldehyde after being cultured for 12 hours. Alexa Fluor 488 Phalloidin (Sigma-Aldrich) and 4',6-diamidino-2-phenylindole (Thermo Fisher Scientific, Waltham, MA, USA) were used to label the F-actin and nuclei of 3T3-E1 cells. The osteoblasts on the samples were observed by laser scanning confocal microscopy (Fv-1000; Olympus, Tokyo, Japan).

\section{Cell proliferation}

To study cell proliferation on different scaffolds, $1 \times 10^{4}$ 3T3-E1 cells were seeded onto BMC, TMC, and NMC scaffold disks $(n=3)$ and cultured in 24-well plates with $1 \mathrm{~mL}$ of culture medium for various culturing intervals (ie, $1,3,5$, and 7 days), and then $10 \mu \mathrm{L}$ per well of Cell Counting Kit-8 (CCK-8; Solarbio, Beijing, People's Republic of China) and $90 \mu \mathrm{L}$ per well of fresh medium were added to the 24-well plates to replace the original culture medium. These plates were then incubated in a $5 \% \mathrm{CO}_{2}$ incubator at $37^{\circ} \mathrm{C}$ for 4 hours. Subsequently, an aliquot of the supernatant solution $(80 \mathrm{~mL})$ was transferred to a 96 -well plate, and the absorbance was measured at $450 \mathrm{~nm}$ using a microplate reader (RT-6000; Rayto, Guangdong, People's Republic of China). Corrected absorbance values were obtained by subtracting the blank absorbance (medium without cells) from the obtained absorbance.

\section{Alkaline phosphatase activity}

To determine the differentiation of 3T3-E1 at different time points, an alkaline phosphatase (ALP) experiment was performed. 3T3-E1 cells seeded onto BMC, TMC, and NMC scaffolds were cultured in DMEM for 3, 5, or 7 days $(n=3)$. ALP activity was determined using an ALP assay kit (NJJCBIO, Nanjing, People's Republic of China).

\section{Animal study}

The Institutional Ethics Committee of the Tianjin Medical University approved the use of SD rats for the experiments conducted in this study. The animal study was performed to evaluate the performances of different scaffolds in vivo. The skull-penetrating defect model of SD rats was established to compare the bone regeneration of different scaffolds. The 8-week-old rats ( $250 \mathrm{~g}$; male) were divided into four groups: 1) control, 2) BMC scaffold, 3) TMC scaffold, and 4) NMC scaffold. The rats were administered general anesthesia via an intraperitoneal injection of chloral hydrate $(1 \mathrm{~mL} / 330 \mathrm{~g}$; 
Yulong, Yangshuo, People's Republic of China). After exposure of the parietal calvarium, a full-thickness skull defect ( $5 \mathrm{~mm}$ in diameter) was prepared with a trephine bur, which was cooled by continuously dripping sterile saline. Then, the different scaffolds ( $5 \mathrm{~mm}$ in diameter) were implanted into the defects, and the incisions were closed with sutures using a 5-0 suture line. In the control group, the defects were sutured without scaffolds.

\section{Micro-computed tomography}

To investigate bone regeneration in situ, micro-computed tomography (CT) scans were performed using a Micro-CT (Sky Scan 1174v2, SkyScan N.V., Kontich, Belgium). The test settings were as follows: each specimen was scanned for 360 rotational steps under an X-ray voltage of $50 \mathrm{kVp}$, anode current of $800 \mu \mathrm{A}$, and exposure time of $1,700 \mathrm{~ms}$. The images were used to reconstruct tomograms with a software package called NRecon.

\section{Histological preparation and evaluation}

The animals were sacrificed by injecting an overdose of pentobarbital sodium at the end of different experimental stages ( 4 weeks and 8 weeks), and then the bone defects with surrounding cranial tissues were fixed in $10 \%$ neutral buffered formalin. Subsequently, specimens were decalcified in a $17 \%$ ethylenediaminetetraacetic acid solution, dehydrated in an ascending graded series of alcohol, and embedded in paraffin. In the center of the bone defects, $5 \mu \mathrm{m}$ sequential transverse sections were performed and stained with hematoxylin and eosin (H\&E) and Masson's trichrome (Baso Diagnostics Inc., Zhuhai, Peoples Republic of China) so that they could be observed by light microscopy (BX51; Olympus).

\section{Statistical analysis}

Statistical analyses were performed using Student's $t$-test. All quantitative experiments were repeated at least three times. Values were expressed as mean \pm standard deviation. Statistical significance was considered at $P<0.05$.

\section{Results}

\section{Micromorphology and characterization of NMC, TMC, and BMC scaffolds}

As shown in Figure $1 \mathrm{~A}-\mathrm{C}$, the collagen molecules extracted from rat tail tendons completed self-assembly into collagen fibrils in $\mathrm{K}_{2} \mathrm{HPO}_{4}$ solution $(0.02 \mathrm{mM})$ and arranged themselves into nanofibers with distinctive cross-bands of 67 $\mathrm{nm}$ periods. Compared to the mineralized scaffolds, fibrils without mineralization had a smaller diameter. The nanofibers of the TMC scaffold (Figure 1B) were the thickest because the larger apatite crystals gathered on the outside of the nanofibers. The apatite crystals made the fibers hard and brittle, and thus, fractures were visible on these fibers. Conversely, the BMC scaffold (Figure 1C) mineralized via the PILP process made the nanoapatites regularly arranged within the collagen fibrils and nanofiber; therefore, the distinctive cross-bands on the nanofibers were more evident. Figure 1D shows the BMC scaffold at low magnification, demonstrating that collagen fibrils interweave into a network with porous microstructures.

To further confirm the level of mineralization, TEM was used to analyze the relationship between collagen fibrils and HA crystals, as shown in Figure 2A-C. In general, fibrils of the collagen scaffold are not visible due to their low material contrast. Figure $2 \mathrm{~A}$ shows the distinctive cross-band of collagen fibrils within the NMC scaffold after being stained by uranyl acetate. Without staining, HA nanocrystals of the BMC scaffold (Figure 2C) depositing inside and near the gap zones of collagen fibrils made the cross-band as clear as being stained. However, the apatite crystals were located outside the fibrils of the TMC scaffold (Figure 2B) because of the large size, which was consistent with the SEM results.

Figure 3 presents the XRD patterns of the three different collagen scaffolds. The curve of the TMC scaffold presented three prominent crystalline peaks at $28.9^{\circ}, 31.7^{\circ}$, and $45.3^{\circ}$ $(2 \theta)$, which were the distinctive diffraction peaks of HA crystals. The curve of the BMC scaffold presented broad and inconspicuous peaks at the same points, indicating that the minerals within the collagen were in the amorphous state. The XRD pattern of the NMC scaffold had no obvious peaks compared with the results of the other two scaffolds.

The results of swelling ratio of different scaffolds at different time points are shown in Figure 4. The swelling ratios of these three different samples are $889 \% \pm 4 \%$ (NMC scaffolds), 972\% $\pm 6 \%$ (BMC scaffolds), and 1,141\% $\pm 6 \%$ (TMC scaffolds). All the swelling degrees exceeded 100\%. The good absorbent characteristic and porous structures of collagen-based scaffolds may be the main reasons of the high swelling property.

Figure 5 shows a comparison of the elastic moduli on different collagen scaffolds under dry and wet conditions. The results showed that mineralization significantly enhanced the elastic moduli of the collagen scaffolds under both wet and dry conditions $(P<0.05)$. Compared with the TMC scaffold (21.64 \pm 2.33$)$, the elastic modulus of the BMC scaffold (41.64 \pm 3.72$)$ clearly increased $(P<0.01)$. Both collagen (NMC) scaffolds and mineralized collagen (TMC and BMC) scaffolds decreased markedly in terms of the elastic modulus under wet conditions $(P<0.01)$, although the BMC scaffolds 

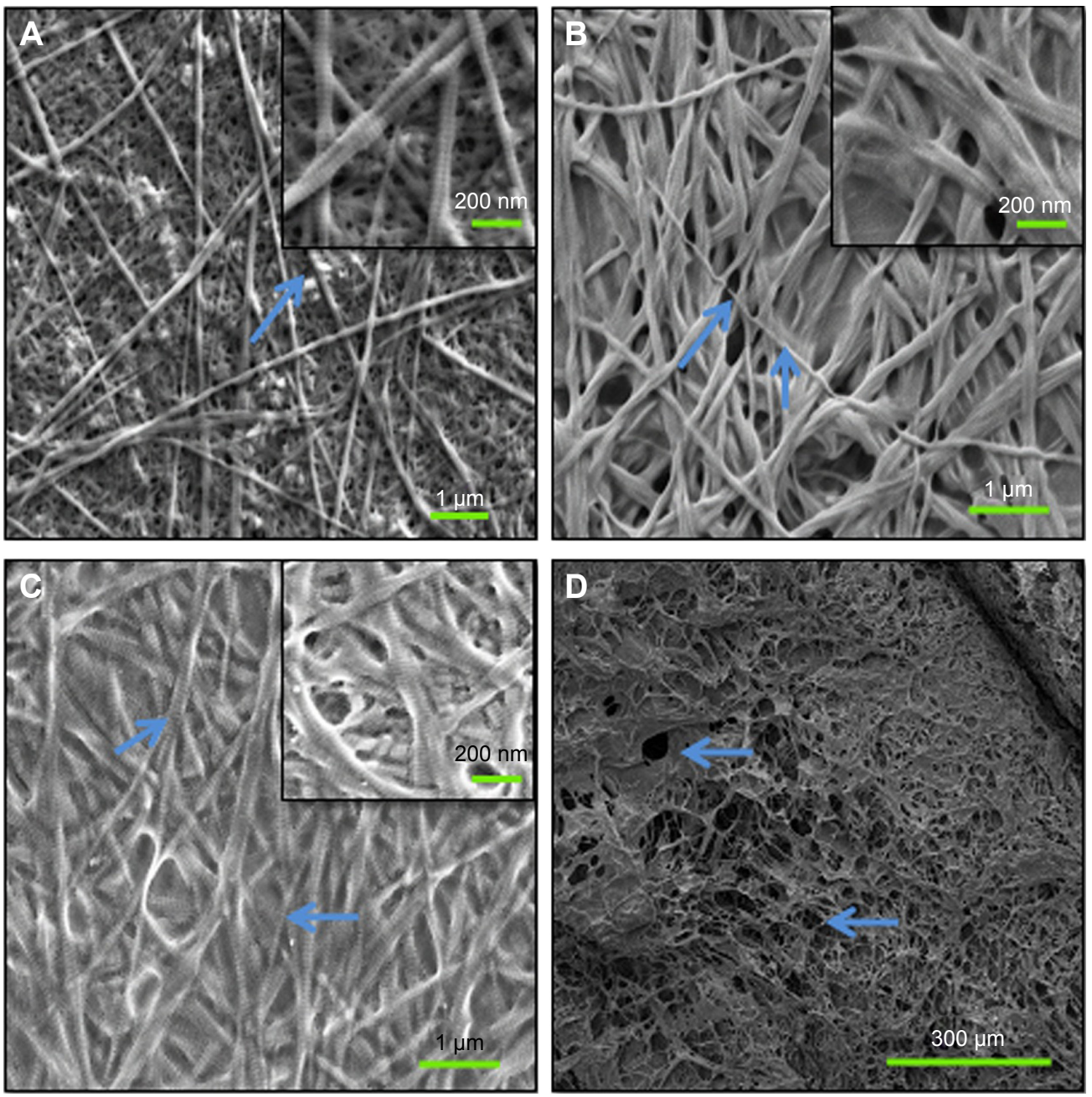

Figure I SEM photographs showing the morphologies of NMC, TMC, and BMC scaffolds.

Notes: (A) NMC scaffold at high magnification; the arrow shows collagen fibrils without mineralization. (B) TMC scaffold at high magnification; the arrows show collagen fibrils mineralized by SBF after 24 hours. (C) BMC scaffold at high magnification; the arrows show collagen fibrils mineralized by CMC/ACP after 24 hours. (D) BMC scaffold at low magnification; the arrows show porous microstructures of BMC scaffold. The upper right illustrations represent local amplification of each photograph, respectively. Abbreviations: ACP, amorphous calcium phosphate; CMC, carboxymethyl chitosan; SBF, simulated body fluid; SEM, scanning electron microscopy; BMC, biomimetic mineralized collagen; NMC, non-mineralized collagen; TMC, traditional mineralized collagen.
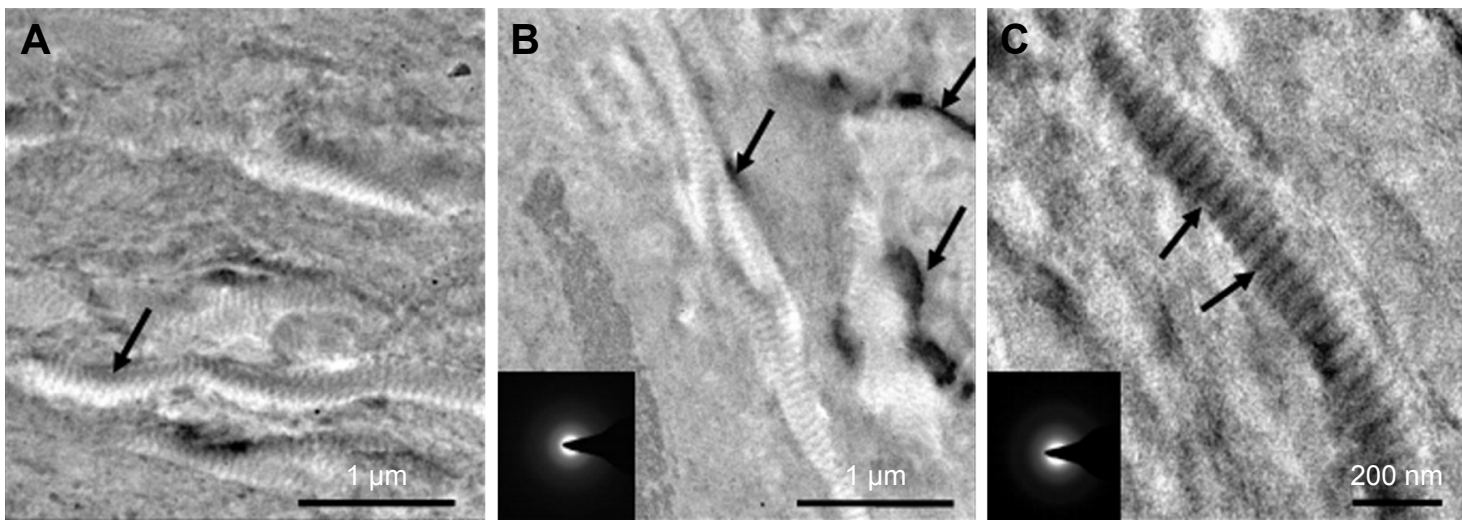

Figure 2 TEM and SAED characterization of NMC, TMC, and BMC scaffolds.

Notes: (A) Image of NMC scaffolds stained by uranyl acetate; the arrow shows distinctive cross-band of collagen fibrils without mineralization. (B) Image of TMC scaffolds unstained; the arrows show the apatite crystals located outside of the fibrils of TMC scaffold. The lower left illustration (SAED) shows obvious ring pattern characteristic of crystal structure. (C) Image of BMC scaffolds without staining; the arrows show HA nanocrystals deposit inside and nearby the gap zones, which make the cross-band quite clear. The lower left illustration (SAED) indicates that the main composition is amorphous phase.

Abbreviations: BMC, biomimetic mineralized collagen; NMC, non-mineralized collagen; TMC, traditional mineralized collagen; HA, hydroxyapatite; SAED, selected-area electron diffraction; TEM, transmission electron microscopy 


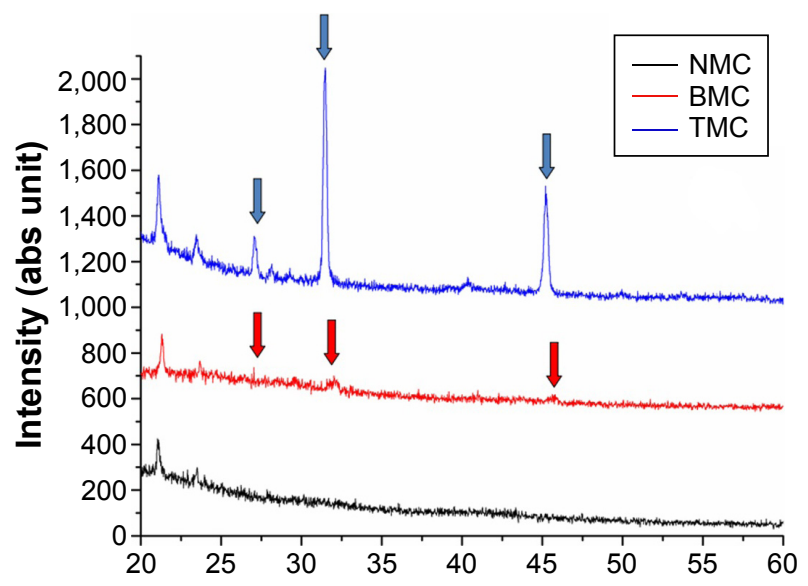

Figure 3 XRD plots for NMC, TMC, and BMC scaffolds.

Note: The distinctive diffraction peaks' sites of HA crystals can be seen as arrows. Abbreviations: BMC, biomimetic mineralized collagen; NMC, non-mineralized collagen; TMC, traditional mineralized collagen; HA, hydroxyapatite; XRD, X-ray diffraction.

still had a significantly higher modulus compared with the other two scaffolds $(P<0.05)$.

The mineral content of each scaffold is shown in Figure 6. The results show that the mineral content of TMC and BMC scaffolds has no statistically significant difference before and after wetting. The trace amount of mineral in NMC scaffolds is likely to come from the inorganic salt solution during the preparation of rat tail tendons from SD rats.

Figure 7 shows the biodegradation of the different scaffolds in vitro. The results reveal that the greatest loss of mass occurred in NMC scaffolds after incubation in PBS with lysozyme. Approximately $80 \%$ of the NMC scaffolds were degraded within 28 days, whereas $\sim 50 \%$ of the mineralized collagen (TMC and BMC) scaffolds were degraded under the same conditions. The degradation rates of BMC scaffolds were significantly slower than those of the other two groups $(P<0.05)$. By contrast, mineralization can significantly

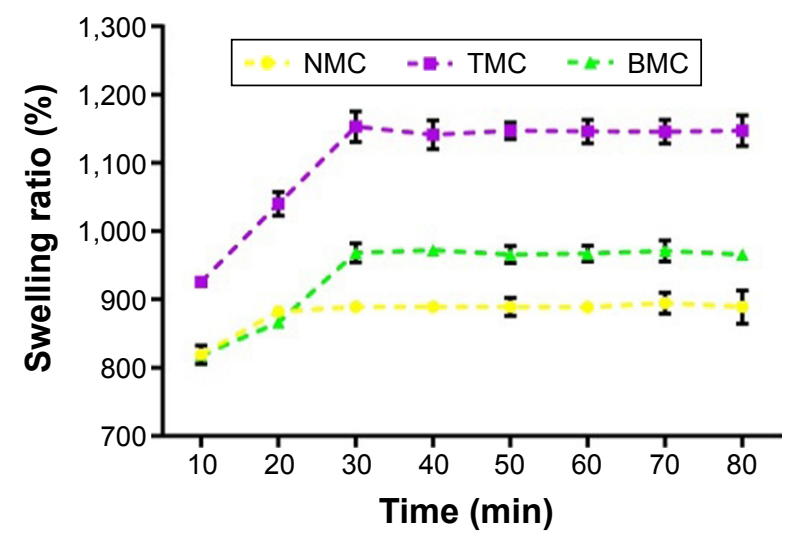

Figure 4 Swelling ratios of NMC, TMC, and BMC scaffolds.

Note: Error bars represent mean \pm SD for $n=3$.

Abbreviations: BMC, biomimetic mineralized collagen; NMC, non-mineralized collagen; TMC, traditional mineralized collagen; SD, standard deviation; min, minutes.

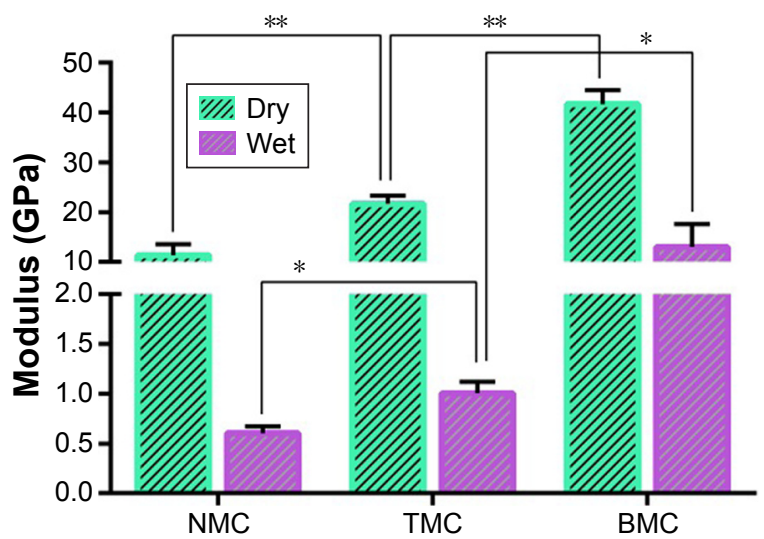

Figure 5 Elastic modulus of NMC, TMC, and BMC scaffolds at dry and wet conditions. Notes: Error bars represent mean $\pm S D$ for $n=4$. In dry conditions, the difference on elastic modulus among three subgroups is statistically significant $(* * P<0.01 ; n=4)$. In wet conditions, the difference is also statistically significant $\left({ }^{*} P<0.05 ; n=4\right)$.

Abbreviations: BMC, biomimetic mineralized collagen; NMC, non-mineralized collagen; TMC, traditional mineralized collagen; SD, standard deviation.

protect collagen from degradation in PBS with lysozyme, especially synergistic intra- and extrafibrillar mineralization, when compared with NMC samples.

\section{Evaluation of biocompatibility in vitro}

The characteristics of 3T3-E1 on the three different scaffolds were evaluated using a laser scanning confocal microscope after being stained by acridine orange/ethidium bromide (Figure 8). There were no significant dead cells (red staining) on all scaffolds after they were cultured for 7 days. This result indicates that all the three different scaffolds exhibit satisfactory cytocompatibility. However, the preosteoblasts on the NMC scaffold maintained a rounded cell morphology and were lesser in number, whereas they were spindle shaped and plentiful on the mineralized collagen scaffolds (Figure 8, column IF) under the same conditions. In contrast with the TMC scaffolds, the

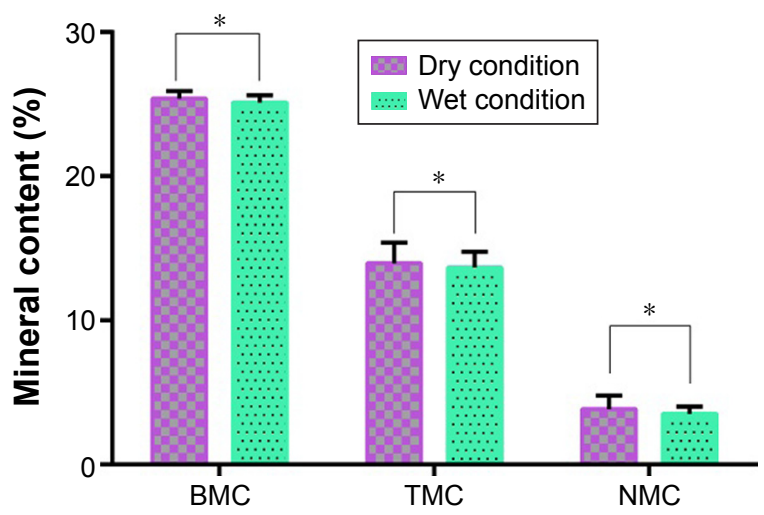

Figure 6 Mineral content of NMC, TMC, and BMC scaffolds at dry and wet conditions.

Notes: Error bars represent mean $\pm S D$ for $n=3$. There is no statistically significant difference on mineral content of different collagen scaffolds before and after wetting $(* P>0.05 ; n=3)$.

Abbreviations: BMC, biomimetic mineralized collagen; NMC, non-mineralized collagen; TMC, traditional mineralized collagen; SD, standard deviation. 


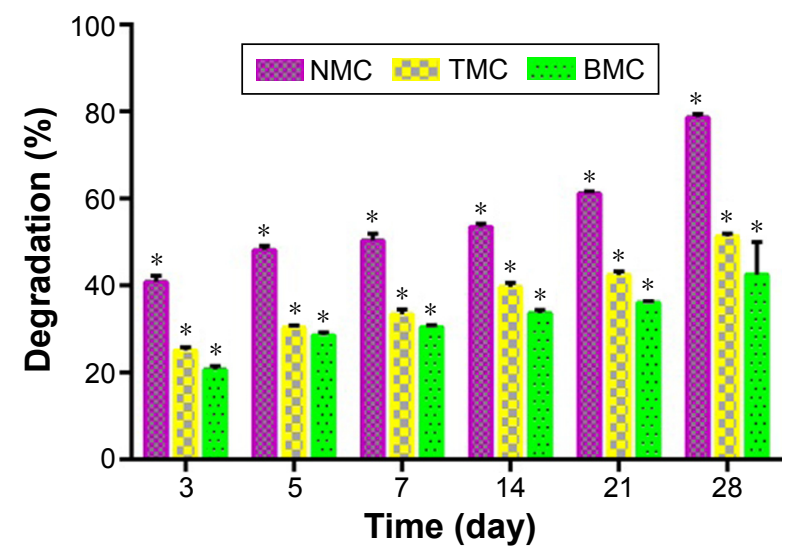

Figure 7 Degradation of NMC, TMC, and BMC scaffolds in vitro.

Notes: Error bars represent mean $\pm S D$ for $n=3$. The difference in degradation among three subgroups is statistically significant ( $* P<0.05$ vs other groups; $n=3$ ).

Abbreviations: BMC, biomimetic mineralized collagen; NMC, non-mineralized collagen; TMC, traditional mineralized collagen; SD, standard deviation.

preosteoblasts on the BMC scaffolds were more spread out, indicating that the BMC scaffolds were more advantageous for the transformation of preosteoblast to osteoblasts.

Figure 9 presents the CCK- 8 assay results of the preosteoblasts seeded on the three different scaffolds after 1, 3, 5, and 7 days. Compared with the NMC and TMC scaffolds, the absorbance of BMC scaffolds exhibits a statistically significant difference at certain time intervals $(P<0.05)$, indicating that the BMC scaffold is beneficial to the growth of preosteoblasts.
The ALP activity of preosteoblasts distributing on different scaffolds at 3, 5, and 7 days is shown in Figure 10. The BMC and TMC scaffolds both presented a significant increase in ALP activity compared with the NMC scaffolds. Although the TMC group exhibited the highest ALP activity at the beginning of the test, the BMC group exhibited clear growth and the highest activity during later testing times. The NMC group always presented the lowest activity during the test phase.

\section{Results of osteogenic potential in vivo}

The diameter of the penetrating defect of the rat skull was $5 \mathrm{~mm}$, as shown in Figure 11. After 4 weeks of implantation, the micro-CT result showed little mineralization in the blank control group (Figure 12A and B) and only a slight amount of mineralization in the TMC group (Figure 12A and $\mathrm{B})$. A certain amount of bone regeneration at the edge of the defect was observed in both the TMC and BMC groups (Figure 12A and B). At 8 weeks, the diameters of the defects in each group had different levels of decrease. Compared with the blank control and NMC groups, the new bone formation in the TMC and BMC groups was more obvious (Figure 12C and $\mathrm{D}$ ). The bone regeneration of the BMC group was the most remarkable of all the groups. These results indicate
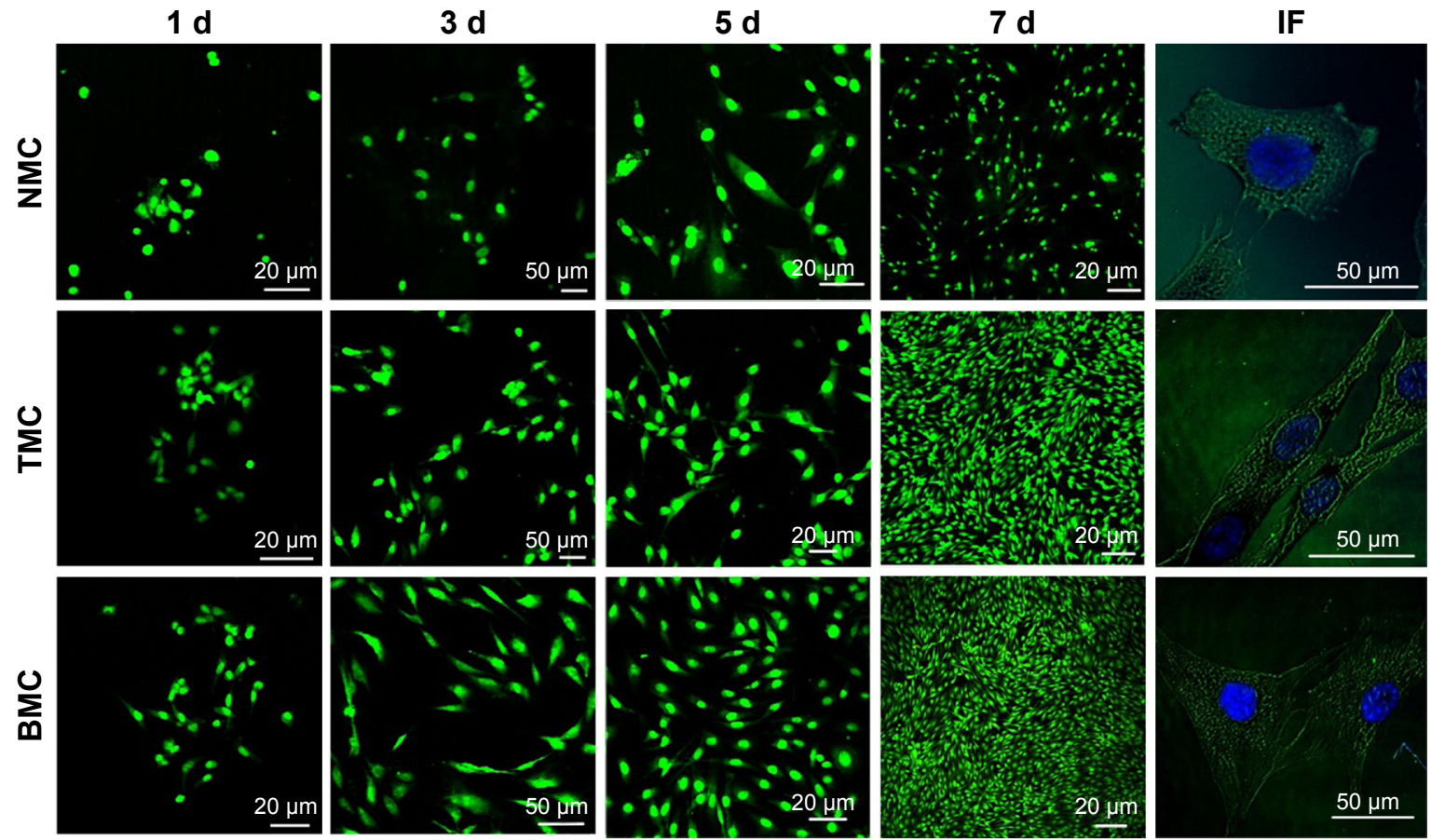

Figure 8 LSCM images of 3T3-EI cells seeded on BMC, TMC, and NMC scaffolds after culturing for I 2 hours (immunocytochemical staining), I, 3 , 5, and 7 days (AO/EB staining). Notes: Column "IF" shows the F-actin cytoskeleton (green) and nuclei (blue) of preosteoblasts grown on different collagen scaffolds.

Abbreviations: AO/EB, acridine orange/ethidium bromide; BMC, biomimetic mineralized collagen; NMC, non-mineralized collagen; TMC, traditional mineralized collagen; d, days; LSCM, Laser scanning confocal microscopy. 


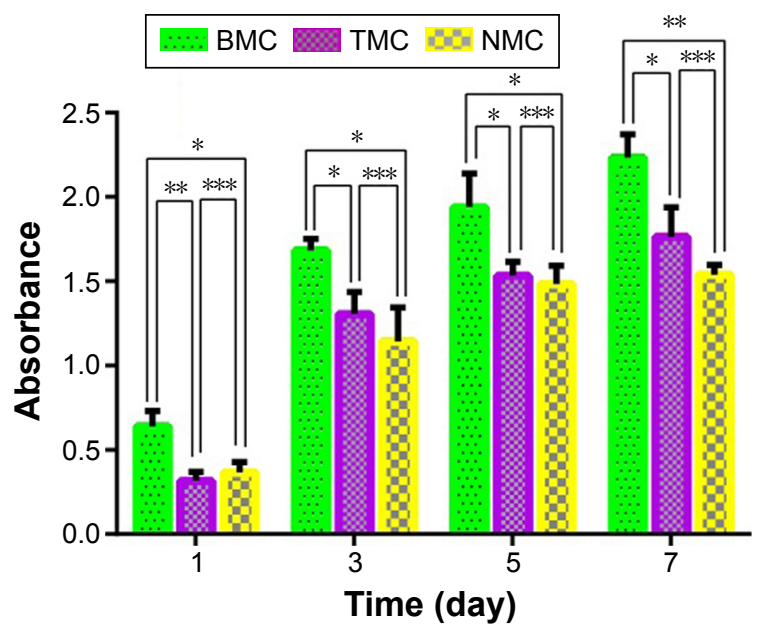

Figure 9 CCK-8 assay results of 3T3-EI cells growing in NMC, TMC, and BMC scaffolds after culturing for I, 3, 5, and 7 days.

Notes: There is a statistically significant difference on cell proliferation between BMC scaffolds and the other two scaffolds at each testing time. The cell proliferation on TMC and NMC scaffolds has no statistical differences at each testing time $(* P<0.05 ; n=3 ; * * P<0.0$ I; $n=3 ; * * * P>0.05 ; n=3)$.

Abbreviations: BMC, biomimetic mineralized collagen; NMC, non-mineralized collagen; TMC, traditional mineralized collagen; CCK-8, Cell Counting Kit-8.

that the BMC scaffolds have a faster bone regeneration rate compared to the other groups.

Figures 13 and 14 show the images of histological sections containing $\mathrm{H} \& \mathrm{E}$ and Masson's trichrome staining. More fibrous connective tissues rather than new bone tissues were observed in the defect of the control group (Figures 13A and B, $14 \mathrm{~A}$ and $\mathrm{B})$. In contrast, more new bone tissues and osteoid appeared in the bone stump in the BMC (Figures 13D and 14D) and TMC (Figures 13C and 14C) groups, particularly in the BMC group. Additionally, at the end of the second month, not only new bone tissues but also marrow organ-like structures appeared in the BMC group (Figures 13D and 14D).

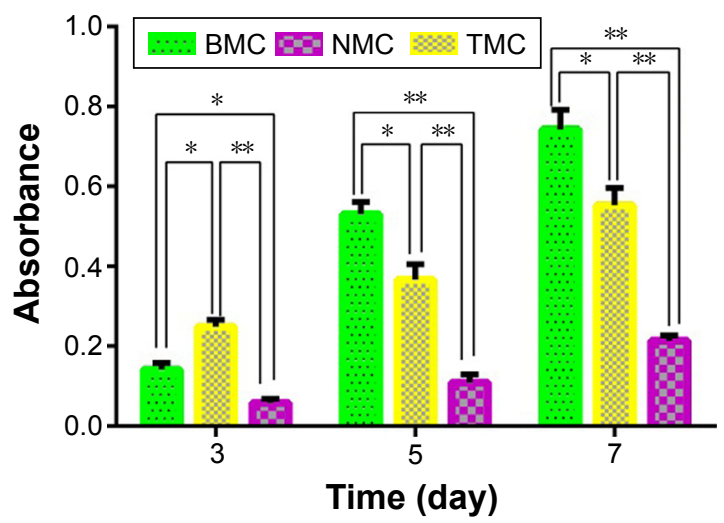

Figure 10 ALP activity of 3T3-EI cells distributing on NMC, TMC, and BMC scaffolds after culturing for 3,5 , and 7 days.

Notes: The difference between BMC scaffolds and the other two scaffolds is statistically significant at each testing time. The ALP activities on TMC and NMC scaffolds are statistically significant at each testing time $\left({ }^{*} P<0.05 ; n=3 ; * * P<0.01 ; n=3\right)$.

Abbreviations: ALP, alkaline phosphatase; BMC, biomimetic mineralized collagen; NMC, non-mineralized collagen; TMC, traditional mineralized collagen.
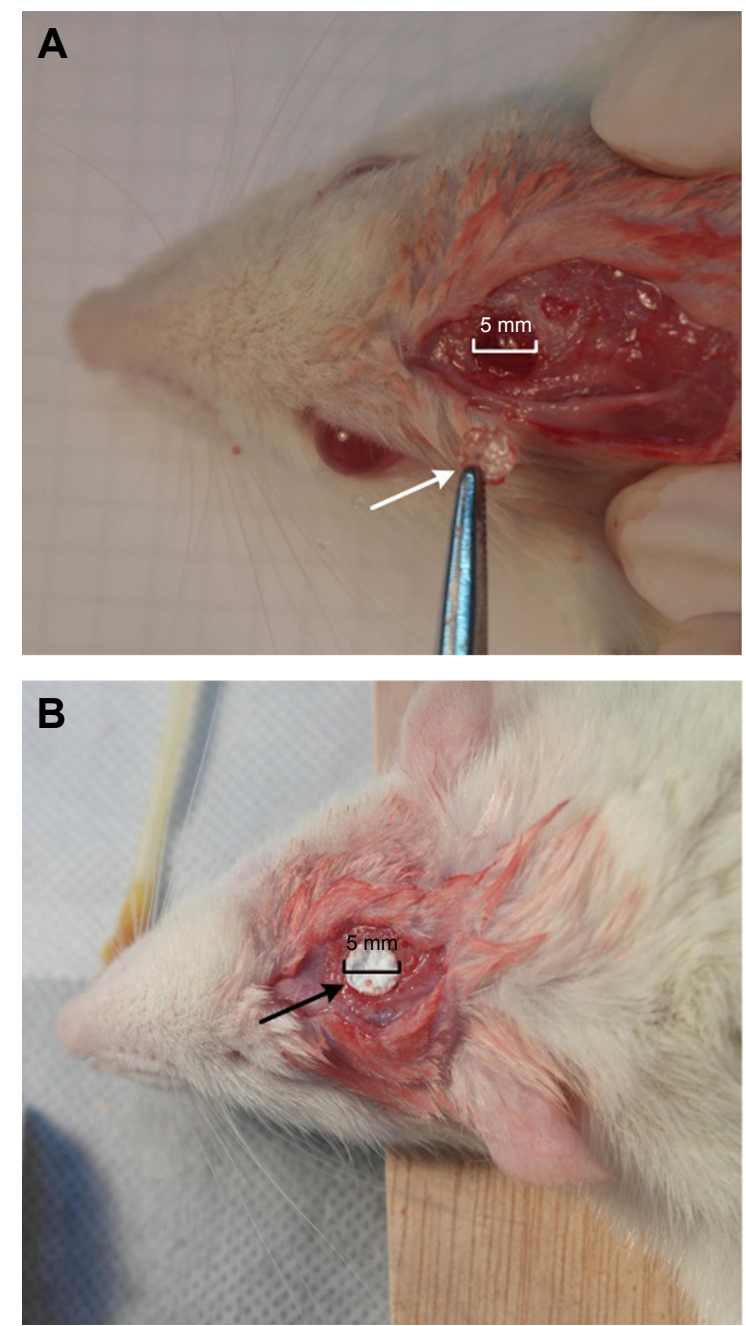

Figure I I Skull-penetrating defect model of SD rats.

Notes: (A) Penetrating defect of SD rat; the arrow shows the bone taken from the skull of SD rat. (B) Scaffold implanted in the defect; the arrow shows the collagen scaffold.

Abbreviation: SD, Sprague-Dawley.

\section{Discussion}

With the progress of testing technology, it has been found that the basic unit of natural bone ECM is mineralized collagen fibrils. ${ }^{14}$ Therefore, collagen has been widely used as the basic element of scaffolds for bone tissue engineering due to its excellent biocompatibility and biodegradability. However, because collagen possesses poor mechanical properties and too high a degradation rate in vivo when used alone, many studies have attempted to modify it through mineralization. ${ }^{1,44-48}$ In the present work, we fabricated a novel synergistically intra- and extrafibrillar mineralized collagen scaffold using precursors of ACP stabilized by CMC, a negatively charged polymer. Type 1 collagen self-assembles into fibrils with a triple-helical molecular structure, which is known as tropocollagen, and thus, we 

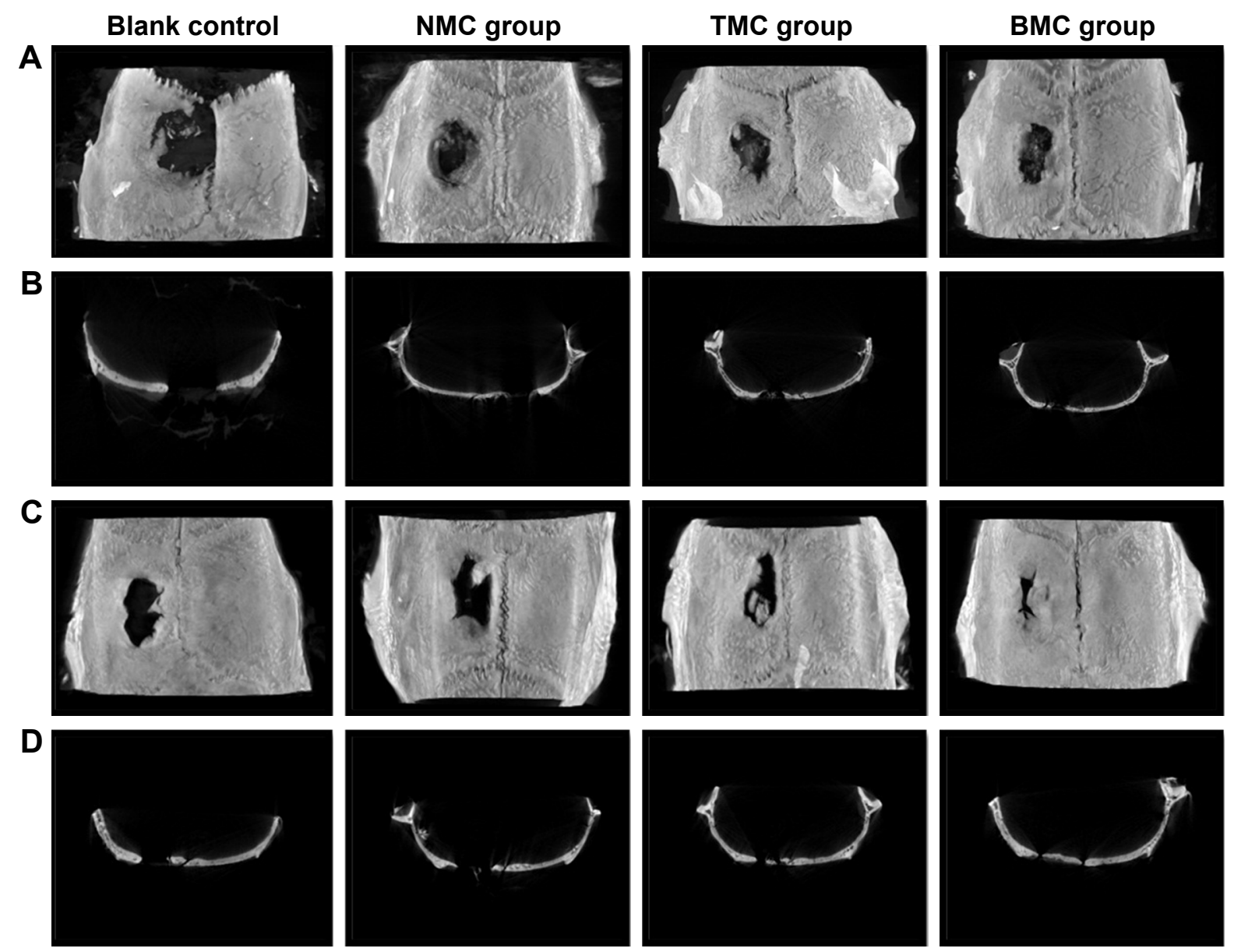

Figure 12 Micro-CT images of new bone formation in each group after 4 and 8 weeks.

Notes: The four rows are reconstructed tomograms of rat craniums after 4 weeks $(\mathbf{A})$ and 8 weeks $(\mathbf{C})$ and coronary sections of rat craniums after 4 weeks (B) and 8 weeks (D).

Abbreviations: BMC, biomimetic mineralized collagen; NMC, non-mineralized collagen; TMC, traditional mineralized collagen; CT, computed tomography.

can see the distinctive cross-band of $67 \mathrm{~nm}$ periods. ${ }^{14}$ Nevertheless, the sizes of HA crystals are considerably larger than the gap zones of collagen fibrils. ${ }^{49,50}$ Hence, we provided a new method for biomimetic mineralization based on the PILP strategy, in which collagen gels after self-assembly, but not dry collagen scaffolds, were immersed into the $\mathrm{CMC} /$ ACP nanocomplex solution. Compared with the direct mixing of collagen solution and mineral ions ${ }^{11,46,47}$ or immersing the dry collagen scaffolds in the mineral solution, ${ }^{13,23,51}$ the mineralized method described in this study can cause the
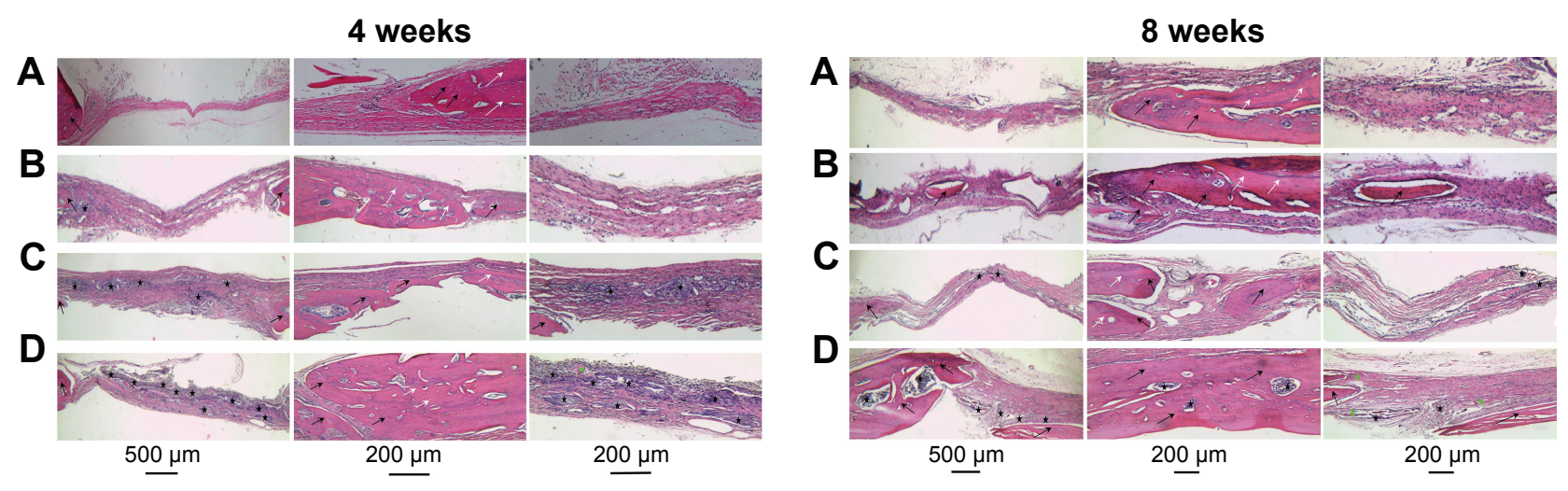

Figure 13 Histological sections (H\&E staining) of rat cranial defect and surrounding tissue at different testing times.

Notes: Blank control (A) NMC scaffolds (B), TMC scaffolds (C), and BMC scaffolds (D). Black arrows, new bone; white arrows, host bone; black stars, scaffold materials; green stars, osteoid.

Abbreviations: BMC, biomimetic mineralized collagen; NMC, non-mineralized collagen; TMC, traditional mineralized collagen; H\&E, hematoxylin and eosin. 

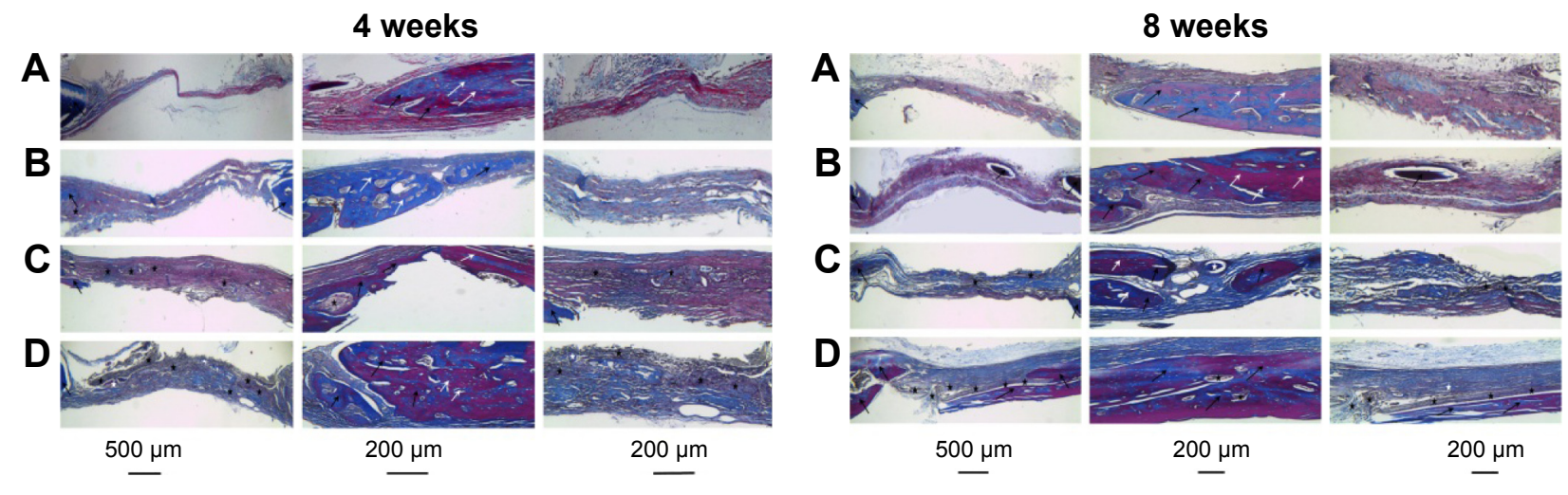

Figure 14 Histological sections (Masson's trichrome staining) of rat cranial defect and surrounding tissue at different testing times: blank control (A), filled with NMC scaffolds (B), filled with TMC scaffolds (C), and filled with BMC scaffolds (D).

Notes: Histological sections (Masson's trichrome staining) of rat cranial defect and surrounding tissue at different testing times: blank control (A), filled with NMC scaffolds (B), filled with TMC scaffolds (C), and filled with BMC scaffolds (D). Black arrows, new bone; white arrows, host bone; black stars, scaffold materials; white star, osteoid. Abbreviations: BMC, biomimetic mineralized collagen; NMC, non-mineralized collagen; TMC, traditional mineralized collagen.

collagen scaffold to have a water oversaturated status after self-assembly. This status may make the nanocomplexes of $\mathrm{CMC} / \mathrm{ACP}$ more easily intercalate into the interstices of the collagen fibrils, which is the crux of intrafibrillar mineralization of collagen scaffolds.

Collagen fibrils extracted from the rat tails after selfassembly, similar to the collagen of natural bone, exhibited distinctive cross-band characteristics. The BMC scaffolds acquired using the bionic method described earlier made the cross-band more obvious in a short period of mineralization (24 hours). During this period, most minerals within the collagen scaffolds were in the amorphous state, which was confirmed by the selected-area electron diffraction results, and the diameters of the BMC fibrils increased because the ACP inside and outside the fibrils transform to HA crystals as time passes. The package of HA also made the collagen fibrils harder, and thus, we can observe traces of fractures on the fibrils under SEM (Figure 1B). The diameter of the $\mathrm{CMC} / \mathrm{ACP}$ nanocomplexes was small enough to make it easy for $\mathrm{CMC} / \mathrm{ACP}$ nanocomplexes to be deposited inside the gap zones of collagen fibrils, which was confirmed by TEM results. In contrast, due to the large sizes of the crystals generated in the SBF, they were only located on the surface of the fibrils (Figure 2B). During the biomimetic mineralization, collagen fibrils are considered to be the primary template for deposition and orientation of the HA nanocrystals. In addition to collagen templates, noncollagenous proteins, particularly DMP-1, are considered to have a close relationship with the mineralization process. DMP-1 has ACP binding sites at one end and collagen binding sites at the other end such that DMP-1 can stabilize the ACP nanoclusters after selfassembly and guide the nanocomplexes into the gap zone of collagen templates. ${ }^{52-54}$ In this study, CMC, as the analog of DMP-1, can stabilize ACP over a proper period of time and guide $\mathrm{CMC} / \mathrm{ACP}$ nanocomplexes into the interior of collagen fibrils. The CMC/ACP nanocomplexes were drawn into the interstices of the collagen fibrils by both the capillary forces of fluidic nature and the interactions between negatively charged nanocomplexes and positive domains in the collagen fibrils (Figure 15), which have been demonstrated by other research groups. ${ }^{53,55}$ After a period of time, the nanocomplexes inside the interstices of the collagen fibrils reached saturation levels such that the other nanocomplexes began to be deposited on the surface of the fibrils. As time passes (72 hours), collagen scaffolds accomplished the synergistic intra- and extrafibrillar mineralization via the transformation of ACP to HA. Therefore, the collagen scaffolds fabricated using this biomimetic method can complete synergistic intra- and extrafibrillar mineralization with the assistance of CMC.

Rapid degradation and poor mechanical properties are the major disadvantages of collagen as scaffolds for bone tissue regeneration. The degradation rate and mechanical properties of scaffolds may affect the long-term success of bone tissue regeneration because the untimely stresses to the new bone are detrimental for the regeneration of tissues. ${ }^{56,57}$ The results in this study prove that the mineralization of collagen can protect collagen from degradation and improve its mechanical properties, which is consistent with other results of other studies. ${ }^{9,58,59}$ To investigate the biological behaviors in vivo of the three different scaffolds, we designed animal experiments and tensile tests in this study. In our animal study, the BMC scaffolds were still visible at the end of 8 weeks after implantation, whereas the NMC scaffolds had almost completely degraded after 4 weeks, which was similar to previous degradation experiments in vitro. ${ }^{9,57}$ The TMC scaffolds can be observed at the end of 4 weeks. However, they almost dispersed after 


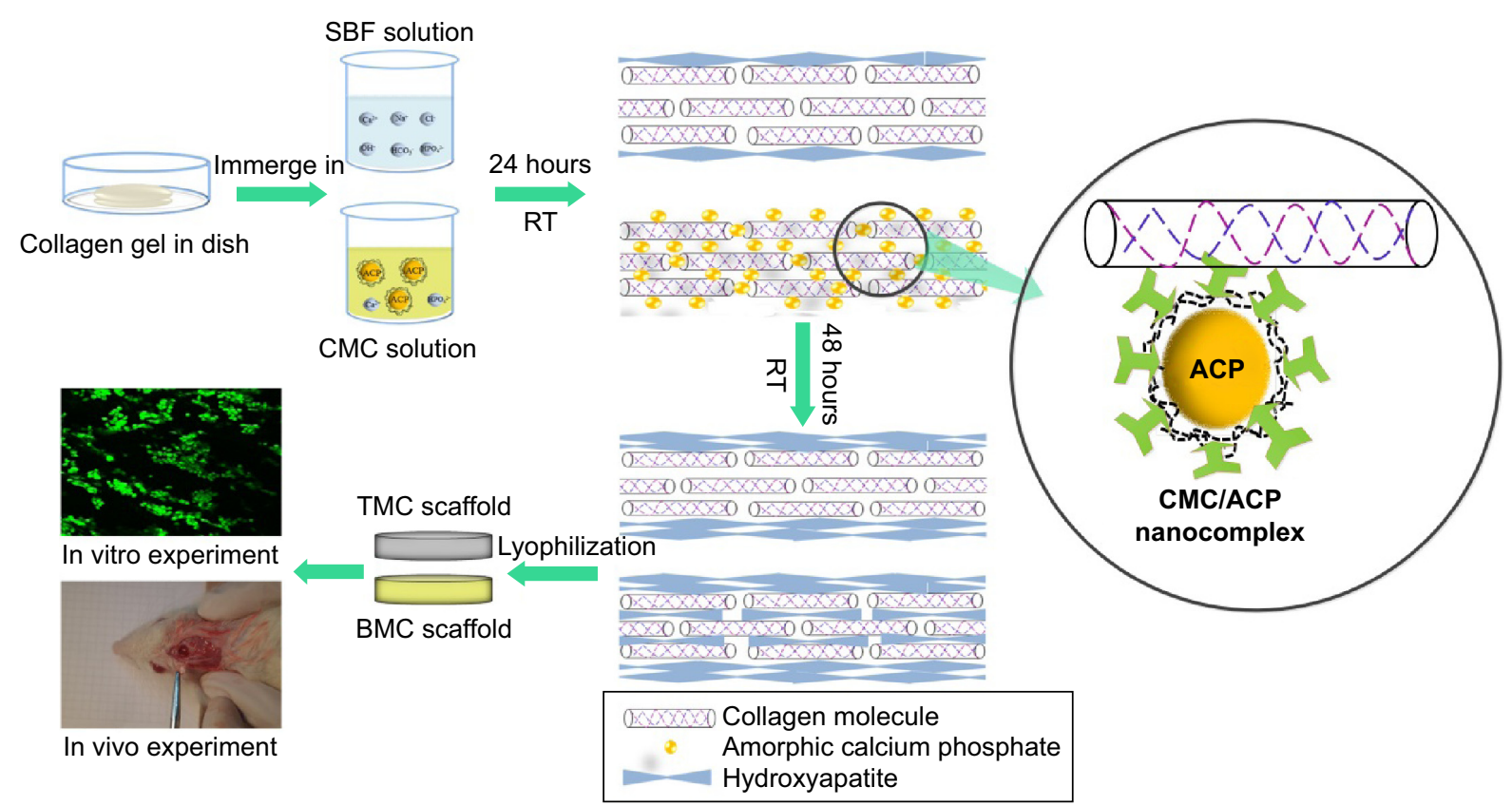

Figure 15 The experimental scheme of synergistic intra- and extrafibrillar mineralization process using CMC/ACP nanocomplexes.

Abbreviations: ACP, amorphous calcium phosphate; BMC, biomimetic mineralized collagen; CMC, carboxymethyl chitosan; RT, room temperature; SBF, simulated body fluid; TMC, traditional mineralized collagen.

8 weeks. Hence, the synergistic intra- and extrafibrillar mineralization can slow the degradation of collagen to a greater degree compared to the extrafibrillar mineralization. The comparison of the elastic modulus among three different scaffolds indicated that the synergistic intra- and extrafibrillar mineralization can improve the mechanical properties of collagen to the maximum extent possible. Compared with the TMC scaffolds, the considerably higher elastic modulus of the BMC scaffolds was primarily attributed to the minerals deposited within the gap zones of the fibrils, similar to natural bone. ${ }^{9,60}$ Because the scaffolds are under wet conditions in vivo, we tested the mechanical properties of the different scaffolds under wet conditions. All the scaffolds had significant reductions in elastic modulus under wet conditions, which was probably caused by the effect of incorporated water. ${ }^{9}$ Despite this condition, the BMC scaffolds still possess the best mechanical properties among all the groups.

Sufficient space and a suitable microenvironment are required for the proliferation and differentiation of cells in vitro. The three-dimensional porous collagen scaffolds with appropriate mechanics, porosity, material composition, concentration, and matrix degradation kinetics will allow cells to be engineered to migrate and spread. However, the micropores of collagen scaffolds without mineralization collapse under the basal medium, which was confirmed by the in vitro studies of 3T3-E1. Because of mineralization, the micropores of TMC and $\mathrm{BMC}$ can be maintained during the process of cell culture such that they have more spaces for cell proliferation, which is similar to the results of another study. ${ }^{57}$ We used 3T3-E1 to examine the osteogenic activity of the three different scaffolds in vitro in the present study. The results of cell proliferation (Figure 9) indicated that intra- and extrafibrillar mineralization can protect the micropores from collapsing to a greater degree compared with the extrafibrillar mineralization, and thus, the cell number on the BMC scaffolds was considerably higher than that on the TMC scaffolds. Another reason for the higher cell number on the BMC scaffold may be due to the biomimetic surface topography and excellent mechanical properties of the BMC scaffolds. ${ }^{9}$ Because ALP is always recognized as one of the most important enzymes produced by osteoblasts, we tested it at different time points as an osteoblastic maker in this study. The level of ALP was obviously higher in the two mineralized groups. This result may be due to the significant effect of mineral HA of osteoblastic differentiation of preosteoblasts (3T3-E1) in vitro. ${ }^{11,61}$ The ALP activity on the TMC scaffolds was slightly higher than that on the BMC scaffolds at the beginning of the test (Figure 10). This may be due to the earlier contacts between 3T3-E1 and HA on the TMC scaffolds while the cells were still proliferating on the BMC scaffolds. However, the ALP activity on the BMC scaffolds caught up with and surpassed that on the TMC scaffolds after a period of time. The nanosized HA within the BMC scaffolds may play an important role during this process, which was 
consistent with the results of other researchers. ${ }^{10,49,62,63}$ All the results of experiments in vitro indicated that the BMC scaffolds can provide more abundant spaces and a more suitable microenvironment to promote the proliferation and differentiation of 3T3-E1.

The animal experiments were used to evaluate the biological behaviors of the three different collagen scaffolds in vivo. The diameter of defects was selected at $5 \mathrm{~mm}$ based on the critical size of defects defined by Schmitz and Hollinger. ${ }^{64}$ The results of micro-CT of specimens (Figure 12) at each time point (4 and 8 weeks) showed that the new bone formation in BMC group was the most marked among all groups at the macro level, indicating that the synergistically intra- and extrafibrillar mineralized scaffolds had good biological compatibility and osteoconductive effect. To observe the differences among the three scaffolds in vivo at the histological level, H\&E and Masson's trichrome stainings were performed, which confirmed the micro-CT results. At the end of 4 weeks, there was almost no residual collagen scaffolds in the NMC group, whereas a considerably greater amount of material could be observed in the TMC and BMC groups, particularly in the latter group. This result indicated that mineralization can protect collagen scaffolds from degrading, particularly synergistic intra- and extrafibrillar mineralization. Hence, the scaffolds can acquire an appropriate degradation rate adapted to the speed of new bone formation such that it is more beneficial to the regeneration of bone tissue in the defects. Furthermore, the osteoid formation with osteoblasts and the initiation of capillary vessels could be well observed at both 4 and 8 weeks in the BMC group, which showed the potential of osteoinduction of the BMC scaffolds. ${ }^{65}$ Masson's trichrome staining of specimens reveals the same results as with $\mathrm{H} \& \mathrm{E}$. The biomimetic BMC scaffolds fabricated in this study present a satisfactory regeneration effect of bone tissue in defects and an ideal degradation rate in vivo. Because the BMC scaffolds acquired using this strategy have satisfactory results both in vitro and in vivo, it has the potential for clinical application for treating bone defects. Due to the abundant sources and low price of chitosan, the scaffolds can be industrially produced in the near future.

\section{Conclusion}

In this study, we fabricated synergistically intra- and extrafibrillar mineralized collagen scaffolds using a new method of biomimetic mineralization in gel form in a CMC/ACP solution based on PILP theory. The results indicated that the BMC scaffold, which was similar to natural bone ECM in terms of chemical composition and microstructure, can better promote the proliferation and differentiation of mouse preosteoblasts and subsequently accelerated the regeneration of bone in the defects of rat calvaria bone compared to the other two groups. Synergistic mineralization can also increase the mechanical strength and decrease the degradation rate of collagen scaffolds at the same time such that the BMC scaffolds can better promote the regeneration of bone tissue in defects.

\section{Acknowledgments}

This study was munificently supported by National Natural Science Foundation of China (Grant numbers 31470920 and 81571016). The authors wish to thank Jichao Wang of Beihang University as well as Shanshan Zhang of Hebei University of Technology for their contributions.

\section{Disclosure}

The authors report no conflicts of interest in this work.

\section{References}

1. Liao SS, Cui FZ, Zhang W, Feng QL. Hierarchically biomimetic bone scaffold materials: nano-HA/collagen/PLA composite. J Biomed Mater Res B Appl Biomater. 2004;69(2):158-165.

2. Niemeyer P, Krause U, Fellenberg J, et al. Evaluation of mineralized collagen and alpha-tricalcium phosphate as scaffolds for tissue engineering of bone using human mesenchymal stem cells. Cells Tissues Organs. 2004;177(2):68-78.

3. Meinel L, Karageorgiou V, Fajardo R, et al. Bone tissue engineering using human mesenchymal stem cells. Ann Biomed Eng. 2004;32(2): 112-122.

4. Wu S, Liu X, Yeung KWK, Liu C, Yang X. Biomimetic porous scaffolds for bone tissue engineering. Mater Sci Eng R Rep. 2014;80:1-36.

5. Stevens MM. Biomaterials for bone tissue engineering. Mater Today. 2008;11(5):18-25.

6. Quinlan E, Thompson EM, Matsiko A, O'Brien FJ, Lopez-Noriega A. Long-term controlled delivery of rhBMP-2 from collagen-hydroxyapatite scaffolds for superior bone tissue regeneration. J Control Release. 2015;207:112-119.

7. Costa-Pinto AR, Correlo VM, Sol PC, et al. Osteogenic differentiation of human bone marrow mesenchymal stem cells seeded on melt based chitosan scaffolds for bone tissue engineering applications. Biomacromolecules. 2009;10(8):2067-2073.

8. Liu Y, Mai S, Li N, et al. Differences between top-down and bottom-up approaches in mineralizing thick, partially demineralized collagen scaffolds. Acta Biomater. 2011;7(4):1742-1751.

9. Liu Y, Luo D, Liu S, et al. Effect of nanostructure of mineralized collagen scaffolds on their physical properties and osteogenic potential. J Biomed Nanotechnol. 2014;10(6):1049-1060.

10. Rodrigues SC, Salgado CL, Sahu A, Garcia MP, Fernandes MH, Monteiro FJ. Preparation and characterization of collagen-nanohydroxyapatite biocomposite scaffolds by cryogelation method for bone tissue engineering applications. J Biomed Mater Res A. 2013;101(4):1080-1094.

11. Minardi S, Corradetti B, Taraballi F, et al. Evaluation of the osteoinductive potential of a bio-inspired scaffold mimicking the osteogenic niche for bone augmentation. Biomaterials. 2015;62:128-137.

12. Jiao K, Niu LN, Li QH, et al. Biphasic silica/apatite co-mineralized collagen scaffolds stimulate osteogenesis and inhibit RANKL-mediated osteoclastogenesis. Acta Biomater. 2015;19:23-32.

13. Antebi B, Cheng X, Harris JN, Gower LB, Chen XD, Ling J. Biomimetic collagen-hydroxyapatite composite fabricated via a novel perfusion-flow mineralization technique. Tissue Eng Part C Methods. 2013;19(7):487-496. 
14. Thula TT, Rodriguez DE, Lee MH, Pendi L, Podschun J, Gower LB. In vitro mineralization of dense collagen substrates: a biomimetic approach toward the development of bone-graft materials. Acta Biomater. 2011;7(8):3158-3169.

15. Ficai A, Andronescu E, Voicu G, et al. Self-assembled collagen/hydroxyapatite composite materials. Chem Eng J. 2010;160(2):794-800.

16. Wang Y, Cui FZ, Hu K, Zhu XD, Fan DD. Bone regeneration by using scaffold based on mineralized recombinant collagen. J Biomed Mater Res B Appl Biomater. 2008;86(1):29-35.

17. Gelinsky M, Welzel PB, Simon P, Bernhardt A, König U. Porous three-dimensional scaffolds made of mineralised collagen: preparation and properties of a biomimetic nanocomposite material for tissue engineering of bone. Chem Eng J. 2008;137(1):84-96.

18. Gomes PS, Santos JD, Fernandes MH. Cell-induced response by tetracyclines on human bone marrow colonized hydroxyapatite and Bonelike. Acta Biomater. 2008;4(3):630-637.

19. Vani R, Girija EK, Elayaraja K, Prakash Parthiban S, Kesavamoorthy R, Narayana Kalkura S. Hydrothermal synthesis of porous triphasic hydroxyapatite/(alpha and beta) tricalcium phosphate. J Mater Sci Mater Med. 2009;20(Supp1 1):S43-S48.

20. Xie G, Sun J, Zhong G, Liu C, Wei J. Hydroxyapatite nanoparticles as a controlled-release carrier of BMP-2: absorption and release kinetics in vitro. J Mater Sci Mater Med. 2010;21(6):1875-1880.

21. Zhou C, Ye X, Fan Y, et al. Biomimetic fabrication of a three-level hierarchical calcium phosphate/collagen/hydroxyapatite scaffold for bone tissue engineering. Biofabrication. 2014;6(3):035013.

22. Yokoyama A, Gelinsky M, Kawasaki T, et al. Biomimetic porous scaffolds with high elasticity made from mineralized collagen - an animal study. J Biomed Mater Res B Appl Biomater. 2005;75(2):464-472.

23. Yang HS, La WG, Park J, Kim CS, Im GI, Kim BS. Efficient bone regeneration induced by bone morphogenetic protein-2 released from apatite-coated collagen scaffolds. J Biomater Sci Polym Ed. 2011;23: 1659-1671.

24. Cui F-Z, Li Y, Ge J. Self-assembly of mineralized collagen composites. Mater Sci Eng R Rep. 2007;57(1-6):1-27.

25. Thula TT, Svedlund F, Rodriguez DE, Podschun J, Pendi L, Gower LB. Mimicking the nanostructure of bone: comparison of polymeric processdirecting agents. Polymers. 2011;3(1):10-35.

26. Liu Y, Li N, Qi YP, et al. Intrafibrillar collagen mineralization produced by biomimetic hierarchical nanoapatite assembly. Adv Mater. 2011;23(8):975-980.

27. Liu Y, Kim YK, Dai L, et al. Hierarchical and non-hierarchical mineralisation of collagen. Biomaterials. 2011;32(5):1291-1300.

28. Niederberger M, Colfen H. Oriented attachment and mesocrystals: nonclassical crystallization mechanisms based on nanoparticle assembly. Phys Chem Chem Phys. 2006;8(28):3271-3287.

29. Meldrum FC, Sear RP. Now you see them. Science. 2008;332: 1802-1803.

30. Zhang S. Fabrication of novel biomaterials through molecular selfassembly. Nat Biotechnol. 2003;21(10):1171-1178.

31. Wong T-S, Brough B, Ho C-M. Creation of functional micro/nano systems through top-down and bottom-up approaches. Mol Cell Biomech. 2009;6(1):1.

32. Gower LB, Odom DJ. Deposition of calcium carbonate films by a polymer-induced liquid-precursor (PILP) process. J Cryst Growth. 2000; 210(4):719-734

33. Dai L, Douglas EP, Gower LB. Compositional analysis of a polymer-induced liquid-precursor (PILP) amorphous $\mathrm{CaCO} 3$ phase. J Non-Cryst Solids. 2008;354(17):1845-1854.

34. Rodriguez DE, Thula-Mata T, Toro EJ, et al. Multifunctional role of osteopontin in directing intrafibrillar mineralization of collagen and activation of osteoclasts. Acta Biomater. 2014;10(1):494-507.

35. George A, Veis A. Phosphorylated proteins and control over apatite nucleation, crystal growth, and inhibition. Chem Rev. 2008;108(11): 4670-4693.

36. He G, George A. Dentin matrix protein 1 immobilized on type I collagen fibrils facilitates apatite deposition in vitro. J Biol Chem. 2004; 279(12):11649-11656.
37. Chen Z, Cao S, Wang H, et al. Biomimetic remineralization of demineralized dentine using scaffold of CMC/ACP nanocomplexes in an in vitro tooth model of deep caries. PLoS One. 2015;10(1):19.

38. Zhang X, Li Y, Sun X, et al. Biomimetic remineralization of demineralized enamel with nano-complexes of phosphorylated chitosan and amorphous calcium phosphate. J Mater Sci Mater Med. 2014; 25(12):2619-2628

39. Jee S-S, Thula TT, Gower LB. Development of bone-like composites via the polymer-induced liquid-precursor (PILP) process. Part 1: influence of polymer molecular weight. Acta Biomater. 2010;6(9):3676-3686.

40. Olszta MJ, Cheng X, Jee SS, et al. Bone structure and formation: a new perspective. Mater Sci Eng R Rep. 2007;58(3-5):77-116.

41. Andrew CA, Khor E, Hastings GW. The influence of anionic chitin derivatives on calcium phosphate crystallization. Biomaterials. 1998; 19(14):1309-1316.

42. Shakir M, Jolly R, Khan MS, Iram N, Khan HM. Nano-hydroxyapatite/ chitosan-starch nanocomposite as a novel bone construct: synthesis and in vitro studies. International Journal of Biological Macromolecules. 2015;80:282-292.

43. Price P. Preparation and use of rat-tail collagen. TCA Manual. 1975; 1(1):43-44.

44. Burg KJ, Porter S, Kellam JF. Biomaterial developments for bone tissue engineering. Biomaterials. 2000;21:2347-2359.

45. Du C, Cui FZ, Zhu XD, de Groot K. Three-dimensional nano-HAp/ collagen matrix loading with osteogenic cells in organ culture. J Biomed Mater Res. 1999;44(4):407-415.

46. Ren X, Bischoff D, Weisgerber DW, et al. Osteogenesis on nanoparticulate mineralized collagen scaffolds via autogenous activation of the canonical BMP receptor signaling pathway. Biomaterials. 2015;50: 107-114.

47. Banglmaier RF, Sander EA, VandeVord PJ. Induction and quantification of collagen fiber alignment in a three-dimensional hydroxyapatitecollagen composite scaffold. Acta Biomater. 2015;17:26-35.

48. Marelli B, Ghezzi CE, Mohn D, et al. Accelerated mineralization of dense collagen-nano bioactive glass hybrid gels increases scaffold stiffness and regulates osteoblastic function. Biomaterials. 2011; 32(34):8915-8926.

49. Zhou H, Lee J. Nanoscale hydroxyapatite particles for bone tissue engineering. Acta Biomater. 2011;7(7):2769-2781.

50. Lin K, Chang J. Structure and properties of hydroxyapatite for biomedical applications. In: Mucalo M, editor. Hydroxyapatite (Hap) for Biomedical Applications. Cambridge, UK: Woodhead Publishing; 2015;1:3-19.

51. Teo WE, Liao S, Chan C, Ramakrishna S. Fabrication and characterization of hierarchically organized nanoparticle-reinforced nanofibrous composite scaffolds. Acta Biomater. 2011;7(1):193-202.

52. Landis WJ, Song MJ. Early mineral deposition in calcifying tendon characterized by high voltage electron microscopy and three-dimensional graphic imaging. J Struct Biol. 1991;107(2):116-127.

53. Li Y, Thula TT, Jee S, et al. Biomimetic mineralization of woven bonelike nanocomposites: role of collagen cross-links. Biomacromolecules. 2012;13(1):49-59.

54. He G, Gajjeraman S, Schultz D, et al. Spatially and temporally controlled biomineralization is facilitated by interaction between self-assembled dentin matrix protein 1 and calcium phosphate nuclei in solution. Biochemistry. 2005;44(49):16140-16148.

55. Nudelman F, Pieterse K, George A, et al. The role of collagen in bone apatite formation in the presence of hydroxyapatite nucleation inhibitors. Nat Mater. 2010;9(12):1004-1009.

56. Fini M, Giannini S, Giardino R, et al. Resorbable device for fracture fixation: in vivo degradation and mechanical behaviour. Int J Artif Organs. 1995;18(12):772-776.

57. Liao SS, Cui FZ. In vitro and in vivo degradation of mineralized llagenBased composite scaffold: nanohydroxyapatite/collagen/ poly(L-lactide). Tissue Eng. 2004;10:73-80.

58. Kim YK, Gu L-S, Bryan TE, et al. Mineralisation of reconstituted collagen using polyvinylphosphonic acid/polyacrylic acid templating matrix protein analogues in the presence of calcium, phosphate and hydroxyl ions. Biomaterials. 2010;31(25):6618-6627. 
59. Kim YK, Mai S, Mazzoni A, et al. Biomimetic remineralization as a progressive dehydration mechanism of collagen matrices-implications in the aging of resin-dentin bonds. Acta Biomater. 2010;6(9):3729-3739.

60. Gupta HS, Seto J, Wagermaier W, Zaslansky P, Boesecke P, Fratzl P. Cooperative deformation of mineral and collagen in bone at the nanoscale. Proc Natl Acad Sci U S A. 2006;103(47):17741-17746.

61. Chuenjitkuntaworn B, Inrung W, Damrongsri D, Mekaapiruk K, Supaphol P, Pavasant P. Polycaprolactone/hydroxyapatite composite scaffolds: preparation, characterization, and in vitro and in vivo biological responses of human primary bone cells. J Biomed Mater Res A. 2010;94(1):241-251.

62. Xie XH, Yu XW, Zeng SX, et al. Enhanced osteointegration of orthopaedic implant gradient coating composed of bioactive glass and nanohydroxyapatite. J Mater Sci Mater Med. 2010;21(7):2165-2173.
63. Lai GJ, Shalumon KT, Chen JP. Response of human mesenchymal stem cells to intrafibrillar nanohydroxyapatite content and extrafibrillar nanohydroxyapatite in biomimetic chitosan/silk fibroin/nanohydroxyapatite nanofibrous membrane scaffolds. Int J Nanomedicine. 2015;10: 567-584.

64. Schmitz JP, Hollinger JO. The critical size defect as an experimental model for craniomandibulofacial nonunions. Clin Orthop Relat Res. 1986;205:299-308.

65. Fang D, Seo BM, Liu Y, et al. Transplantation of mesenchymal stem cells is an optimal approach for plastic surgery. Stem Cells. 2007;25(4): 1021-1028.
International Journal of Nanomedicine

\section{Publish your work in this journal}

The International Journal of Nanomedicine is an international, peerreviewed journal focusing on the application of nanotechnology in diagnostics, therapeutics, and drug delivery systems throughout the biomedical field. This journal is indexed on PubMed Central, MedLine, CAS, SciSearch $®$, Current Contents $\AA /$ Clinical Medicine,

\section{Dovepress}

Journal Citation Reports/Science Edition, EMBase, Scopus and the Elsevier Bibliographic databases. The manuscript management system is completely online and includes a very quick and fair peer-review system, which is all easy to use. Visit http://www.dovepress.com/ testimonials.php to read real quotes from published authors.

Submit your manuscript here: http://www.dovepress.com/international-journal-of-nanomedicine-journal 